\title{
The Effects of Dilazep and SG 75 on Cyclic Nucleotides in the Coronary Artery
}

\author{
Akira Kobayashi, M.D., Kouichi Ogawa, M.D. \\ and Noboru Yamazaki, M.D.
}

\section{SUMMARY}

We examined the effects of new long acting coronary vasodilators (dilazep and SG 75) on cyclic nucleotides in the coronary artery and the myocardium at the maximum coronary blood flow. The cyclic AMP concentrations in the anterior descending coronary artery after the injection of saline (control), SG $75(0.2 \mathrm{mg} / \mathrm{Kg}$ ), and dilazep $(0.1 \mathrm{mg} /$ $\mathrm{Kg}$ ) were $229 \pm 20 \mathrm{pmole} / \mathrm{Gm}, 249 \pm 21 \mathrm{pmole} / \mathrm{Gm}$, and $320 \pm 21 \mathrm{pmol} /$ $\mathrm{Gm}$, respectively. A significant increase above control values was found in the dilazep treated group $(\mathrm{p}<0.02)$. The cyclic GMP concentrations in the coronary artery after the injection of saline, SG 75, and dilazep were $35.6 \pm 4.4 \mathrm{pmole} / \mathrm{Gm}, 40.4 \pm 3.3 \mathrm{pmole} / \mathrm{Gm}, 35.3 \pm 3.2 \mathrm{pmole} / \mathrm{Gm}$, respectively. There were no significant differences between them. The cyclic AMP and cyclic GMP concentrations in the left ventricular muscle did not significantly increase after the administration of dilazep and SG 75. Our findings showed that the mechanism of coronary vasodilating action of dilazep might be associated with an increased cyclic AMP in the coronary artery.

\section{Additional Indexing Words:}

Microwave radiation Cyclic AMP Cyclic GMP Dilazep SG

75 Coronary artery Left ventricular muscle Radioimmunoassay

$I^{1}$ $T$ is well known that there is a close relationship between coronary blood flow and myocardial metabolism. The substances or factors which have been proposed as playing a role in linking metabolic activity and vascular tone are oxygen, carbon dioxide, $\mathrm{H}^{+}, \mathrm{K}^{+}$, lactic acid, increased osmolarity, prostaglandin, adenosine, adenine nucleotides, and others. ${ }^{11}$

Many recent reports have suggested that adenosine cyclic $3^{\prime}, 5^{\prime}$-monophosphate (cyclic AMP) and guanosine cyclic 3',5'-monophosphate (cyclic GMP) play an important role in smooth muscle tone. ${ }^{2-4)}$ Cyclic AMP and cyclic GMP are biological effectors as second messengers involved in regulating cellular functions. We have measured the cyclic AMP and cyclic GMP

From the Second Department of Internal Medicine, Nagoya University School of Medicine, Tsuruma-cho, Showa-ku, Nagoya 466, Japan.

Received for publication March 13, 1979.

Manuscript revised May 23, 1979. 
concentrations in the canine coronary arteries and left ventricular muscle after the administration of various coronary vasodilators in vivo and classified them into 3 groups in association with the effects on cyclic nucleotides in coronary artery. ${ }^{5 !}$

The 1,4-bis[3-(3,4,5-trimethoxybenzoyloxy) propyl] perhydroi, 4-dilazepine (dilazep) and $\mathrm{N}$-(2-hydroxyethyl)nicotinamid nitrate (SG 75) are new coronary vasodilators. The mechanisms of the vasodilating action of both agents have not yet been fully explored.

We examined the effects of dilazep and SG 75 on cyclic AMP and cyclic GMP in the coronary artery and myocardjum of dogs to clarify the relationship between cyclic nucleotides and coronary vasodilating action of these agents.

\section{Materials and Methods}

Thirty-one adult heparinized mongrel dogs weighing $10-12 \mathrm{Kg}$ were anesthetized with sodium pentobarbital (30 mg/Kg I.V.). An endotracheal tube was applied to each dog and artificial respiration was performed with room air with a Harvard respirator. Thoracotomy was performed on the 4th or 5th intercostal space and the pericardium was opened. After $30 \mathrm{~min}$, dilazep $(0.1 \mathrm{mg} / \mathrm{Kg})$, SG $75(0.2 \mathrm{mg} / \mathrm{Kg})$, and saline were injected into the left external jugular vein. Dilazep was a gift from Cowa Pharmaceutical Co and SG 75 was from Chugai Phamaceutical Co. Physiological saline was administered in control group. A polyethylene cannula was introduced into the coronary sinus through the right external jugular vein and it was placed at almost wedged position under fluoroscopic control. The blood from the coronary sinus was returned to the left external jugular vein and an electromagnetic flowmeter (Nihon-Koden MF-2) was placed between the coronary sinus and the left external jugular vein. The coronary blood outflow was measured continuously.

When the coronary blood outflow was at its maximum after the administration of agent, the heart was removed and immediately irradiated for 2 min with microwave $(2450 \mathrm{MHz}, 600 \mathrm{~W})$ for the inactivation of the enzymes. After the irradiation, the left anterior descending coronary artery branch and the left ventricular muscle were removed from the heart and weighed in cold room at $4^{\circ} \mathrm{C}$. Fifty $\mathrm{mg}$ of tissue samples were collected and were homogenized in $1 \mathrm{ml} \mathrm{of} 6 \%$ trichloroacetic acid in ice-cold water bath. The homogenatez were centrifuged at 3,000 rpm for $15 \mathrm{~min}$ at $4^{\circ} \mathrm{C}$ and the supernatant removed and extracted 3 times with $5 \mathrm{ml}$ of ethyl ether saturated with water. The extracted aqueous phase was evaporated with a freezed-dry evaporator and then stored at $-20^{\circ} \mathrm{C}$ until assay of the concentrations of cyclic AMP and cyclic GMP.

The concentrations of cyclic AMP and cyclic GMP were measured by radioimmunoassay of Steiner et $\mathbf{a l}^{6 /}$ with some modification ${ }^{7)}$ using an assay kit of Yamasa. To $100 \mu \mathrm{l}$ of sample was added $100 \mu \mathrm{l}$ of the dioxane-triethylamine mixture containing succinic anhydride. After $10 \mathrm{~min}$ at room temperature, the reaction mixture was added with $0.5 \mathrm{ml}$ of $0.5 \mathrm{M}$ imidazole buffer which contained $0.5 \%$ 
bovine serum albumin, $8 \mathrm{mM}$ theophylline, and $0.01 \%$ streptomycin. To $100 \mu \mathrm{l}$ of the above mixture was added $100 \mu \mathrm{l}$ of $\left[{ }^{125} \mathrm{I}\right]$ succinyl cyclic AMP tyrosine methyl ester or $\left[{ }^{125} \mathrm{I}\right]$ succinyl cyclic GMP tyrosine methyl ester $(15,000-20,000 \mathrm{cpm}$ in an amount less than $10^{-14}$ mole) and $100 \mu \mathrm{l}$ of the diluted antisera; the mixture was kept at $4^{\circ} \mathrm{C}$ overnight (about $20 \mathrm{hr}$ ). A cold solution of dextran-coated charcoal $(0.5 \mathrm{ml})$ was added to the above mixture cooled in an ice-cold water bath. Charcoal was then spun down, and $0.5 \mathrm{ml}$ of the supernatant was counted for radioactivity in a gamma spectrometer.

Mean and standard error of the mean were calculated and statistical analysis was made by using Student's unpaired " $t$ " test. The $p$ values of less than 0.05 were considered significant.

\section{Results}

I. The effects on the coronary blood flow of SG 75 and dilazep.

As shown in Fig. 1, SG 75 in a dose of $0.2 \mathrm{mg} / \mathrm{Kg}$ caused an increase in the coronary blood outflow and its maximum was at 30 to $60 \mathrm{sec}$ after the injection from the left external jugular vein. The duration of the increase

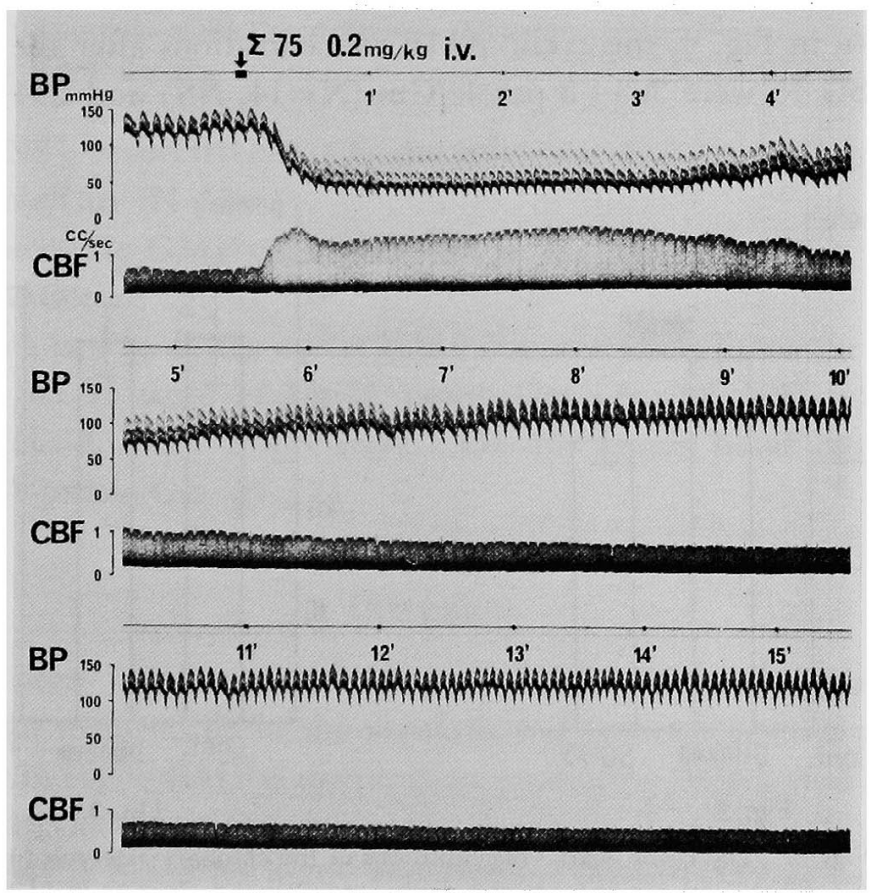

Fig. 1. The effects of SG $75(0.2 \mathrm{mg} / \mathrm{kg} \mathrm{I.V.)}$ on the coronary blood flow (CBF) and blood pressure (BP). SG 75 significantly increased the conary blood flow and at the same time decreased the blood pressure. The maximum increase of the coronary blood flow was at about $30 \mathrm{sec}$ after the injection. BP: Blood pressure CBF: Coronary blood flow 
in the coronary blood outflow caused by SG 75 was about $10 \mathrm{~min}$. On the other hand, dilazep in a dose of $0.1 \mathrm{mg} / \mathrm{Kg}$ caused an increase in the coronary blood outflow and its maximum was 2 to $5 \mathrm{~min}$ after the injection from the left external jugular vein.

II. The cyclic AMP concentrations in the coronary artery at the maximum coronary blood flow after the administration of agents.

As shown in Fig. 2, the cyclic AMP concentrations after the injection of dilazep and SG 75 were $320 \pm 21$ pmole $/ \mathrm{Gm}(\mathrm{N}=14, \mathrm{p}<0.02)$ and $249 \pm$ $21 \mathrm{pmole} / \mathrm{Gm}(\mathrm{N}=10$, NS) respectively, while, it was $229 \pm 20 \mathrm{pmole} / \mathrm{Gm}$ $(\mathrm{N}=7)$ in control group.

III. The cyclic GMP concentrations in the coronary artery at the maximum coronary blood flow after the administration of agents.

As shown in Fig. 3, the cyclic GMP concentrations after the injection of dilazep and SG 75 were $35.3 \pm 3.2$ pmole $/ \mathrm{Gm}(\mathrm{N}=14$, NS) and $40.4 \pm$ $3.3 \mathrm{pmole} / \mathrm{Gm}(\mathrm{N}=10, \mathrm{NS})$, respectively. But, it was $35.6 \pm 4.4 \mathrm{pmole} / \mathrm{Gm}$ $(\mathrm{N}=7)$ in control group.

IV. The cyclic AMP concentrations in the left ventricular muscle after the administration of agents.

As shown in Fig. 4, the cyclic AMP concentrations after the injection of dilazep and SG 75 were $303 \pm 8$ pmole $/ \mathrm{Gm}(\mathrm{N}=14, \mathrm{NS})$ and $294 \pm 38 \mathrm{pmole} /$

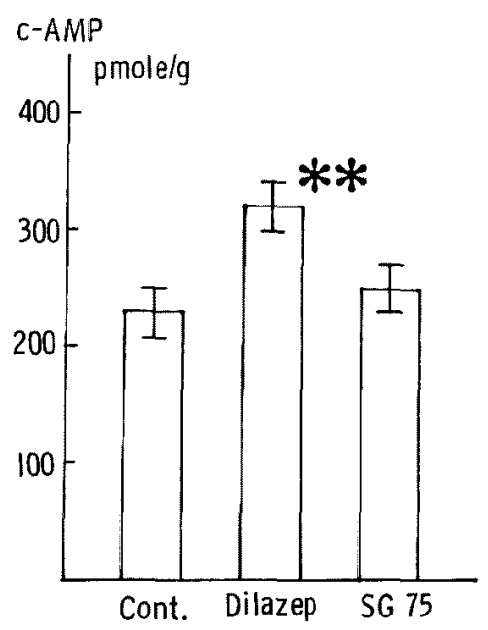

Fig. 2.

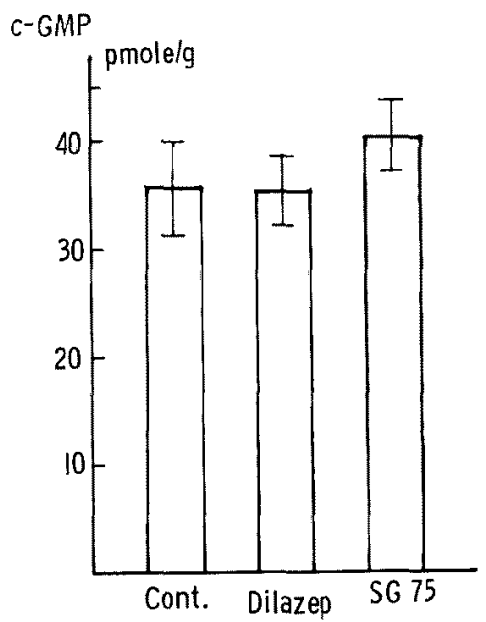

Fig. 3.

Fig. 2. The cyclic AMP concentrations in the coronary artery at maximum increase of the coronary blood flow after the injection of SG $75(0.2 \mathrm{mg} / \mathrm{kg}$ I.V.) and dilazep $(0.1 \mathrm{mg} / \mathrm{kg} \mathrm{I.V.).} \mathrm{Control} \mathrm{group} \mathrm{was} \mathrm{administered} \mathrm{saline.}$ Cont.: Control group ** $\mathrm{p}<0.02$

Fig. 3. The cyclic GMP concentrations in the coronary artery at maximum increase of the coronary blood flow after the injection of SG 75 and dilazep. There were no significant differences. Cont.: Control group 


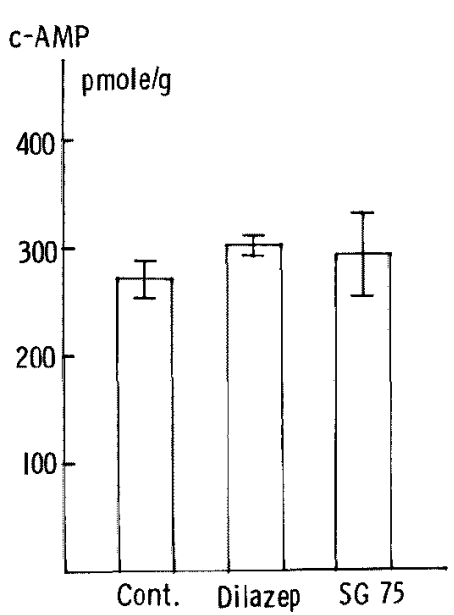

Fig. 4.

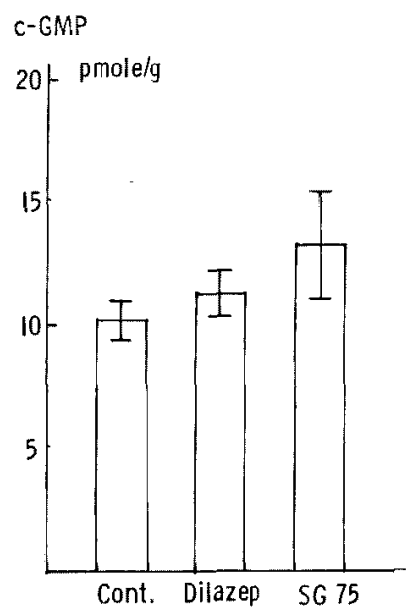

Fig. 5.

Fig. 4. The cyclic AMP concentrations in the left ventricular muscle after the injection of SG 75 and dilazep. There were slight increases of cyclic AMP after the injection of both agents, but these increases were not significant. Cont: Control group

Fig. 5. The cyclic GMP concentrations in the left ventricular muscle after the injection of SG 75 and dilazep. There were no significant differences. Cont.: Control group

$\mathrm{Gm}(\mathrm{N}=8, \mathrm{NS})$, respectively. On the other hand, in control group, it was $272 \pm 16$ pmole $/ \mathrm{Gm}(\mathrm{N}=7)$.

V. The cyclic GMP concentrations in the left ventricular muscle after the administration of agents.

As shown in Fig. 5, the cyclic GMP concentrations after the injection of dilazep and SG 75 were $11.3 \pm 0.9 \mathrm{pmole} / \mathrm{Gm}(\mathrm{N}=14, \mathrm{NS})$ and $13.3 \pm 2.2$ pmole $/ \mathrm{Gm}$ ( $\mathrm{N}=8$, NS), respectively. On the other hand, control group was $10.2 \pm 0.8 \mathrm{pmole} / \mathrm{Gm}(\mathrm{N}=7)$.

\section{Discussion}

It has been suggested that changes in tissue concentration of cyclic nucleotides act reciprocally in the regulation of vascular smooth muscle tone, with increase in cyclic GMP concentration promoting contraction and increase in cyclic AMP concentration promoting relaxation of vascular smooth muscle. This hypothesis is based on many reports that cyclic GMP of vascular strips can be increased by various agents, known to be capable of contracting vascular smooth muscle and cyclci AMP can be increased by a variety of vasodilator drugs. ${ }^{8), 9)}$ But many recent reports do not support the previously suggested role for the cyclic nucleotides in the control of smooth muscle 
motility. ${ }^{10)-12}$. Thus, the relationships between cyclic AMP and cyclic GMP in the smooth muscle during contraction and relaxation are complex.

When cyclic AMP and cyclic GMP concentrations of tissue are measured, most methods of fixing tissue samples have been carried out in liquid nitrogen. During the administration of drugs the changes in cyclic AMP and cyclic GMP concentrations in a small region may be masked by massive amount of non-responding tissue if the cyclic nucleotides concentrations are measured in the whole organ. But, following freezing in liquid nitrogen, the coronary artery can not be dissected easily from the heart. Therefore, this technique do not apply to this study.

A new approach to the problem of rapid tissue fixation and accurate dissection of tissue sample was investigated. A microwave irradiation was employed to rapidly inactivate brain adenyl cyclase and phosphodiesterase and thereby the measurement of cyclic AMP concentration in discrete brain areas could be made possible. ${ }^{13}$ )

Then, we have extended this technique to measure cyclic AMP and cyclic GMP concentrations in the coronary artery. Our previous findings that the cyclic AMP and cyclic GMP concentrations in the left ventricular muscle remained constant following microwave irradiation $(2450 \mathrm{MHz}$, $600 \mathrm{~W}$ ) for $30 \mathrm{sec}$ proved sufficient to inactivate the synthetic and degradative enzymes. ${ }^{51}$

In this study, we measured the cyclic AMP and cyclic GMP concentrations after the administration of dilazep and SG 75. These results indicated that after the injection of dilazep $(0.1 \mathrm{mg} / \mathrm{Kg})$ the cyclic AMP concentrations in the coronary artery significantly increased $(\mathrm{p}<0.02)$ but the injection of SG $75(0.2 \mathrm{mg} / \mathrm{Kg})$ did not increase them. On the other hand, the cyclic GMP concentrations in the coronary artery did not significantly increase after the injection of dilazep and SG 75. As a result, dilazep increased the ratio of cyclic AMP to cyclic GMP but SG 75 did not change it.

We have studied the effects of various coronary vasodilators on cyclic AMP and cyclic GMP concentrations in the canine coronary artery and classified them into 3 groups according to the effect on the ratio of cyclic AMP to cyclic GMP. Group I, including papaverine, dipyridamole, and amyl nitrite, increased the ratio. Group II, including nitroglycerin and isosorbide dinitrate, decreased the ratio. Group II, including nifedipine and diltiazem, did not change the ratio (Table I). From these results, dilazep was included into group I and SG 75 was included into group III according to our classification.

It is suggested that the smooth muscle relaxant effects of papaverine and dipyridamole are likely to be mediated by an increase in cyclic $\mathrm{AMP}^{3}$ ) and 
Table I. The relation of cyclic AMP and cyclic GMP in the coronary artery after the administration of coronary vasodilators

\begin{tabular}{c|l|c|c|c}
\hline Group & Coronary vasodilator & c-AMP & c-GMP & c-AMP/c-GMP \\
\hline \multirow{4}{*}{ I } & papaverin hydrochloride & $\uparrow$ & $\uparrow$ & $\uparrow$ \\
& Dipyridamole & $\uparrow$ & $\nearrow$ & $\uparrow$ \\
& Amyl nitrite & $\nearrow$ & - & $\uparrow$ \\
\hline \multirow{2}{*}{ II } & Nitroglycerin & - & $\uparrow$ & $\downarrow$ \\
\hline \multirow{2}{*}{ III } & Isosorbide dinitrate & - & $\uparrow$ & $\downarrow$ \\
\hline \multirow{2}{*}{ I } & Nifedipine & - & - & - \\
\hline III & Diltiazem hydrochloride & - & - & - \\
\hline
\end{tabular}

Marks of column of cyclic AMP and cyclic GMP signify those that follow. $\uparrow$ and $\uparrow$ show significant increase and an increase of $\uparrow$ is more larger than that of $\uparrow . \nearrow$ shows slight increase and - shows unchanged. On the other hand, $\uparrow$ of column of cyclic AMP/cyclic GMP shows an increase of the ratio of cyclic AMP to cyclic GMP. \ of column of cyclic AMP cyclic GMP/shows a decrease and - shows unchanged of the ratio of cyclic AMP to cyclic GMP.

the mechanism of relaxing effect of cyclic AMP has been hypothesized that the increased cyclic AMP stimulates calcium uptake of sarcoplasmic reticulum by cyclic AMP-dependent protein kinase. ${ }^{14)}$

Berne hypothesized that adenosine was always released in a small amount from the myocardium and the amount of this adenosine release was increased in hypoxic condition and metabolic regulation of coronary circulation was mediated by adenosine. ${ }^{15)}$ Adenosine produces a powerful coronary vasodilation. Sano postulated that dilazep and dipyridamole enhanced coronary vasodilating action of adenosine and its mechanism was mainly due to the decreased disappearance of adenosine from blood. ${ }^{16)}$ Sattin and Roll have reported that adenosine and adenine nucleotides increase in the cyclic AMP concentration of guinea pig cerebral cortex slices. ${ }^{17}$ ) Yoshida et al also recently have observed that the cyclic AMP concentration in the canine coronary artery significantly increases after the administration of ATP and adenosine. ${ }^{18)}$ Although the mechanism whereby adenosine increases cyclic AMP concentration is still in doubt, Clark et al have hypothesized that adenosine increases intracellular cyclic AMP concentration as a result of its interraction with a specific plasma membrane-surface receptor which results in the activation of adenylate cyclase. ${ }^{19)}$ It is suggested that papaverine and dipyridamole increase cyclic AMP concentration as a result of inhibition of phosphodiesterase. From this study, it is uncertain whether dilazep has a stimulatory 
effect on adenylate cyclase activity or an inhibitory effect on phosphodiesterase activity. This result, e.g. the cyclic AMP concentration increased after the injection of dilazep at the maximum coronary blood flow, suggests that the mechanism of coronary vasodilating action of dilazep may be mediated by an increased cyclic AMP in the coronary artery smooth muscle as dipyridamole and papaverine.

On the other hand, SG 75 had no effect on the cyclic AMP and cyclic GMP concentrations in the coronary artery at its maximum dilatation. SG 75 was included into group III as nifedipine and diltiazem, which were calcium antagonist. This result indicated that the mechanism of coronary vasodilating action of SG 75 might be likely to be caused by a different mechanism from cyclic AMP and cyclic GMP.

Recently, the evidence for a close relation between cyclic AMP and ventricular arrhythmias was provided by some experiments that increased tissue cyclic AMP concentration was found in ischemic rat hearts perfused with free fatty acid or pyruvate and that the perfusion of isolated rat heart with dibutyryl cyclic AMP lowered the ventricular fibrillation threshold and widened the vulnerable period. ${ }^{201,21)}$ Opie has postulated that a large amount of accumulation of cyclic AMP in the ischemic heart may predispose to ventricular fibrillation. ${ }^{22}$ It seems that the most desirable coronary vasodilator has no effect on the cyclic AMP concentration in the ventricular muscle from the point of risk of the occurrence of ventricular arrhythmias.

Then, we investigated the effects on the cyclic AMP concentration in the left ventricular muscle after the injection dilazep and SG 75. There were slight increases in cyclic AMP concentration after the injection of both agents, but these increases were not statistically significant. However, in our previous findings, papaverine and dipyridamole significantly increased the cyclic AMP concentration in the left ventricular muscle. From the effects on the cyclic AMP in the left ventricular muscle, dilazep and SG 75 may be clinically more desirable coronary vasodilators.

\section{ConcLusion}

We investigated the relation between cyclic nucleotides in the coronary artery and coronary vasodilating action of dilazep and SG 75. From this study, it seems that the mechanism of coronary vasodilating action of dilazep may be associated with an increased cyclic AMP in the coronary artery. On the other hand, SG 75 had no effect on the cyclic AMP and cyclic GMP in the coronary artery. Dilazep and SG 75 did not significantly increase cyclic AMP concentration in the left ventricular muscle. Thus, both agents are 
more desirable coronary vasodilator drugs from the point of risk of the occurrence of ventricular arrhythmias.

\section{REFERENCES}

1. Braunwald E, Ross J, Sonnenblick EH: Mechanism of Contraction of Normal and Failing Heart. 2nd Ed, Little, Brown \& Co, Boston, p200-231, 1976

2. Goldberg ND, Haddox MK, Nicol SE, Glass DB, Sanford CH, Kuehl FA, Estensen R: Biologic regulation through opposing influences of cyclic GMP and cyclic AMP. in The Yin Yang Hypothesis. Adv Cyclic Nucleotide Res. Raven Press, New York, p307-330, 1975

3. Pöch G, Kukovetz WR: Studies on the possible role of cyclic AMP in drug-induced coronary vasodilatation. in Adv Cyclic Nucleotide Res. Raven Press, New York, p195-211, 1972

4. Diamond J: Role of cyclic nucleotides in control of smooth muscle contraction. in Adv. Cyclic Nucleotide Res. Raven Press, New York, p327-340, 1978

5. Kobayashi A, Ogawa $\mathbf{K}$ : The effects of coronary vasodilator on cyclic nucleotides. Jap Circulat J 43: 46 (Suppl 1), 1979 (in Japanese)

6. Steiner AL, Parker CW, Kipnis DM: Radioimmunoassay for cyclic nucleotides I. Preparation of antibodies and iodinated cyclic nucleotides. J Biol Chem 247: 1106, 1972

7. Honma M, Satoh T, Takezawa J, Ui M: An ultrasensitive method for the simultaneous determination of cyclic AMP and cyclic GMP in small-volume samples from blood and tissue. Biochem Med 18: 257, 1977

8. Rasmussen H, Goodman DBP: Relationships between calcium and cyclic nucleotides in cell activation. Physiol Rev 57: 424, 1977

9. Beam KG, Nestler EJ, Greengard P: Increased cyclic GMP levels associated with contraction in muscle fibers of the giant barnacle. Nature 267: 534, 1977

10. Katsuki S, Murad F: Regulation of adenosine cyclic 3',5'-monophosphate and guanosine cyclic 3',5'-monophosphate levels and contractility in bovine tracheal smooth muscle. Mol Pharmacol 13: 330, 1977

11. Diamond J, Blisard KS: Effects of stimulant and relaxant drugs on tension and cyclic nucleotide levels in canine femoral artery. Mol Pharmacol 12:688, 1976

12. Schultz KD, Schultz K, Schultz G: Sodium nitroprusside and other smooth muscle-relaxants increase cyclic GMP levels in rat ductus deferns. Nature 265: 750, 1977

13. Schmidt MJ, Schmidt DE, Robison GA: Cyclic adenosine monophosphate in brain areas. Microwave irradiation as a means of tissue fixation. Science 173: 1142, 1971

14. Tada M, Kirchberger MA, Katz AM: Phosphorylation of a 22,000-dalton component of the cardiac sarcoplasmic reticulum by adenosine $3^{\prime}: 5^{\prime}$-monophosphate-dependent protein kinase. J Biol Chem 250: 2640, 1975

15. Berne RM: Cardiac nucleotides in hypoxia: possible role in regulation of coronary blood flow. Am J Physiol 204: 317, 1963

16. Sano N: Enhancement of coronary vasodilating action of adenosine by dilazep and dipyridamole in the dog. Jap J Pharmacol 24: 471, 1974

17. Sattin A, Rall TW: The effect of adenosine and adenine nucleotides on the cyclic adenosine 3',5'-monophosphate content of guinea pig cerebral cortex slices. Mol Pharmacol 6: 13, 1970

18. Yoshida M, Ogawa K, Yamazaki N: The effects of ATP on the cyclic AMP of the coronary artery. Kiso to Rinsho 12: 602, 1978 (in Japanese)

19. Clark RB, Gross R: Regulation of adenosine $3^{\prime}, 5^{\prime}$-monophosphate content in human astrocytoma cells by adenosine and the adenine nucleotides. J Biol Chem 249: 5296, 1974

20. Lubbe WF, Bircknell OL, Podzuweit T, Opie LH: Cyclic AMP as a determinant of vulnerability to ventricular fibrillation in the isolated rat heart. Cardiovasc Res 10: 697,1976

21. Sugiura M, Ogawa K, Yamazaki N: Concentration of myocardial cyclic AMP and ventricu- 
lar fibrillation induced by aminophylline. Jap Heart J 20: 177, 1979

22. Podzuweit T, Dalby AJ, Cherry GW, Opie LH: Cyclic AMP levels in ischaemic and nonischaemic myocardium following coronary artery ligation. Relation to ventricular fibrillation. J Mol Cell Cardiol 10: 81, 1978 